\title{
Safety of pediatric procedural sedation in a Canadian emergency department
}

\author{
Urbain Ip, MD; Anurag Saincher, MD
}

\begin{abstract}
Objective: To assess the safety of pediatric procedural sedation performed by emergency physicians working within a structured sedation protocol.

Methods: A retrospective review of all children undergoing emergency department (ED) procedural sedation during a 2-year period after the institution of a structured sedation protocol.

Results: 167 children underwent procedural sedation, primarily for orthopedic manipulation, wound management and foreign body removal. Of these, $82 \%$ received ketamine, $17 \%$ received fentanyl and midazolam and 1\% received midazolam alone. Sedation was adequate in all but 6 patients, who required supplemental ketamine for orthopedic manipulation. Vomiting after arousal occurred in 17 children (10\%), but no episodes of clinical aspiration occurred. One child became agitated during recovery and another experienced a transient visual hallucination. There were no cases of laryngospasm, apnea or cardiorespiratory compromise, and no mortality or significant morbidity occurred.

Conclusion: Emergency physicians using a structured sedation protocol can safely perform ED pediatric procedural sedation. Where intravenous access is not already present, intramuscular ketamine, administered in the doses described, is a safe and effective agent for pediatric sedation.

\section{RÉSUMÉ ANALYTIQUE}

Objectif : Évaluer la sécurité de la sédation avant une intervention douloureuse chez l'enfant par un urgentologue respectant un protocole de sédation structuré.

Méthodes: Revue rétrospective des dossiers de tous les enfants ayant été soumis à une sédation avant une intervention douloureuse à l'urgence au cours d'une période de 2 ans suivant l'établissement d'un protocole de sédation structuré.

Résultats : Cent soixante-sept enfants subirent une sédation avant une intervention, principalement pour une manipulation orthopédique, le traitement d'une blessure et l'excision d'un corps étranger. Parmi ceux-ci, $82 \%$ reçurent de la kétamine, $17 \%$ reçurent du fentanyl ainsi que du midazolam et $1 \%$ reçurent du midazolam seul. La sédation était adéquate chez tous les patients, sauf chez six d'entre eux qui durent recevoir une dose supplémentaire de kétamine pour une manipulation orthopédique. Dix-sept enfants (10\%) furent victimes de vomissements après leur réveil, mais il n'y eut aucun épisode d'aspiration clinique. Un enfant devint agité pendant la récupération et un autre fut en proie à des hallucinations visuelles transitoires. II n'y eut aucun cas de laryngospasme, d'apnée ni de détresse cardiorespiratoire. II n'y eut aucun décès ni morbidité importante.

Conclusions : Les urgentologues qui suivent un protocole de sédation structuré peuvent effectuer la sédation avant une intervention douloureuse chez l'enfant à l'urgence en toute sécurité. En l'absence d'un accès intraveineux, la kétamine intramusculaire, administrée aux doses recommandées, est un agent sécuritaire et efficace pour effectuer la sédation chez l'enfant.
\end{abstract}

Key words: procedural sedation, conscious sedation, pediatric, ketamine, emergency department 


\section{Introduction}

Procedural sedation of adults for brief painful procedures is commonly performed in North American emergency departments (ED). US centres have published extensive data documenting effectiveness, safety and a low incidence of easily-managed complications during pediatric sedation. ${ }^{1-10}$ In Canada, however, pediatric procedural sedation by non-anesthesiologists is controversial and poorly described in the literature.

Given the apparent safety of this procedure, as documented in several large studies, a pediatric procedural sedation protocol was devised and implemented at the Surrey Memorial Hospital (SMH). To evaluate the safety of this protocol, we collected data prospectively for 2 years, then reviewed our experience with 167 children.

\section{Methods}

\section{Design}

A retrospective review of prospectively gathered sedation data.

\section{Setting}

A suburban community hospital ED with an annual census of 78,000 patients, including 26,000 under the age of 17 .

\section{Subjects}

Patients from 6 months to 16 years were eligible for the procedural sedation protocol if they underwent brief sedation for a painful procedure between Jan. 1, 1996, and Dec. 31, 1997, and if they were considered ASA class I as defined in the American Society of Anesthesiologists physical status classification. Patients were excluded if they had poor airway anatomy that would preclude active airway management.

\section{Interventions}

Eligible patients and caregivers were advised about the nature of the procedure, the side effects of medications and the likelihood of complications. Baseline demographic and clinical data were recorded, and verbal informed consent was obtained. No attempt was made to influence the clinicians' choice of sedating agents; however, when ketamine was selected, physicians were required to complete a specific ketamine eligibility form. Figure 1 summarizes the SMH sedation protocol.

\section{Figure 1. Procedural Sedation Protocol}

- Contraindications to procedural sedation are identified.

- The attending physician explains to patient and caregivers the need for the procedure, the effects of medications being used, and the associated risks. Verbal consent is obtained.

- The patient is placed on NPO status. The ED nurse documents a presedation assessment on the sedation record (Fig. 2).

- The patient is connected to an ECG monitor, oxygen saturation monitor and automated blood pressure monitor. Oxygen is applied by mask or nasal cannula.

- The resuscitation cart is brought to the bedside. Oral airway, bag-valve-mask, suction, and reversal drugs are made immediately available.

- The respiratory technologist, nurse and attending ED physician remain at the bedside.

- Medications are administered. The choice of agent and route of administration is at the discretion of the attending physician.

- Vital signs are recorded every 5 minutes during the procedure and for 15 minutes after. Vital signs are then recorded every 15 minutes until discharge criteria are met. One-to-one nursing care is maintained during the monitoring period.

- Readiness for discharge is assessed according to the discharge criteria key (Fig. 2). Patients must achieve a score of 7 prior to discharge.

- The entire procedure is documented on the procedural sedation record.

- Written and verbal after-care instructions* are given to the patient's caregiver prior to discharge.

- If the agent chosen is ketamine, the ED physician must complete a specific ketamine eligibility form to assure appropriate use.

*Available from the authors on request. 
Using specific procedural sedation data forms (Fig. 2), dedicated bedside nurses documented (in real-time) the procedure performed, medication dose and route, ongoing vital signs, level of consciousness, discharge criteria, critical times, and adverse events. A copy of each sedation record was reviewed by the authors within a few days to assure protocol compliance, safety and full data collection. Telephone follow-up was attempted in all cases to identify late adverse effects and determine parental satisfaction with the procedure.

\section{Data analysis}

Descriptive statistics were presented for patient demographics, procedures performed, medication, dose and route used, adverse reactions, time of emergency presentation and duration of ED stay.

\section{Results}

During the 2-year period, 167 children underwent procedural sedation, including 103 males (62\%) and 64 females $(38 \%)$. All charts were available for review and none were excluded from analysis. Table 1 shows that patients ranged in age from 8 months to 16 years and that most $(71 \%)$ were less than 4 years old. Overall, 22 patients $(13 \%)$ presented between 0700 and $1500 \mathrm{~h}, 137(82 \%)$ between 1500 and $0200 \mathrm{~h}$, and $8(5 \%)$ between 0200 and $0700 \mathrm{~h}$.

Table 2 shows that orthopedic manipulation and wound repair were the most common indications for sedation. In children less than 12 months old, indications included sedation for CT scan, ophthalmologic exam, laceration repair, orthopedic manipulation and dorsal slit procedure for paraphimosis.

\begin{tabular}{lcr}
\multicolumn{3}{|c}{ Table 1. Age distribution } \\
\hline Age range & \multicolumn{1}{c}{$n$} & $\%$ \\
\hline 6 mo to $1 \mathrm{yr}$ & 27 & 16 \\
1 to $4 \mathrm{yr}$ & 92 & 55 \\
4 to $8 \mathrm{yr}$ & 31 & 18 \\
8 to $12 \mathrm{yr}$ & 11 & 7 \\
12 to $16 \mathrm{yr}$ & 6 & 4 \\
\hline Total & 167 & 100 \\
\hline
\end{tabular}

Table 3 shows that 133 (79\%) of 167 children received intramuscular (IM) ketamine. When vascular access had been obtained for other reasons, the combination of intravenous (IV) fentanyl and midazolam was most often administered (28 cases). IV ketamine was administered in only 5 cases.

Sedation was adequate in all but 6 children, who required supplemental IM ketamine $(1-3 \mathrm{mg} / \mathrm{kg})$ for orthopedic manipulation. The mean time interval from drug administration to discharge eligibility was 65.2 minutes.

Of 138 patients who received ketamine, 50 (36\%) developed nausea on arousal and 17 (12\%) vomited. Most parents observed transient gait unsteadiness during the recovery period, lasting 1-2 hours. No episodes of laryngospasm

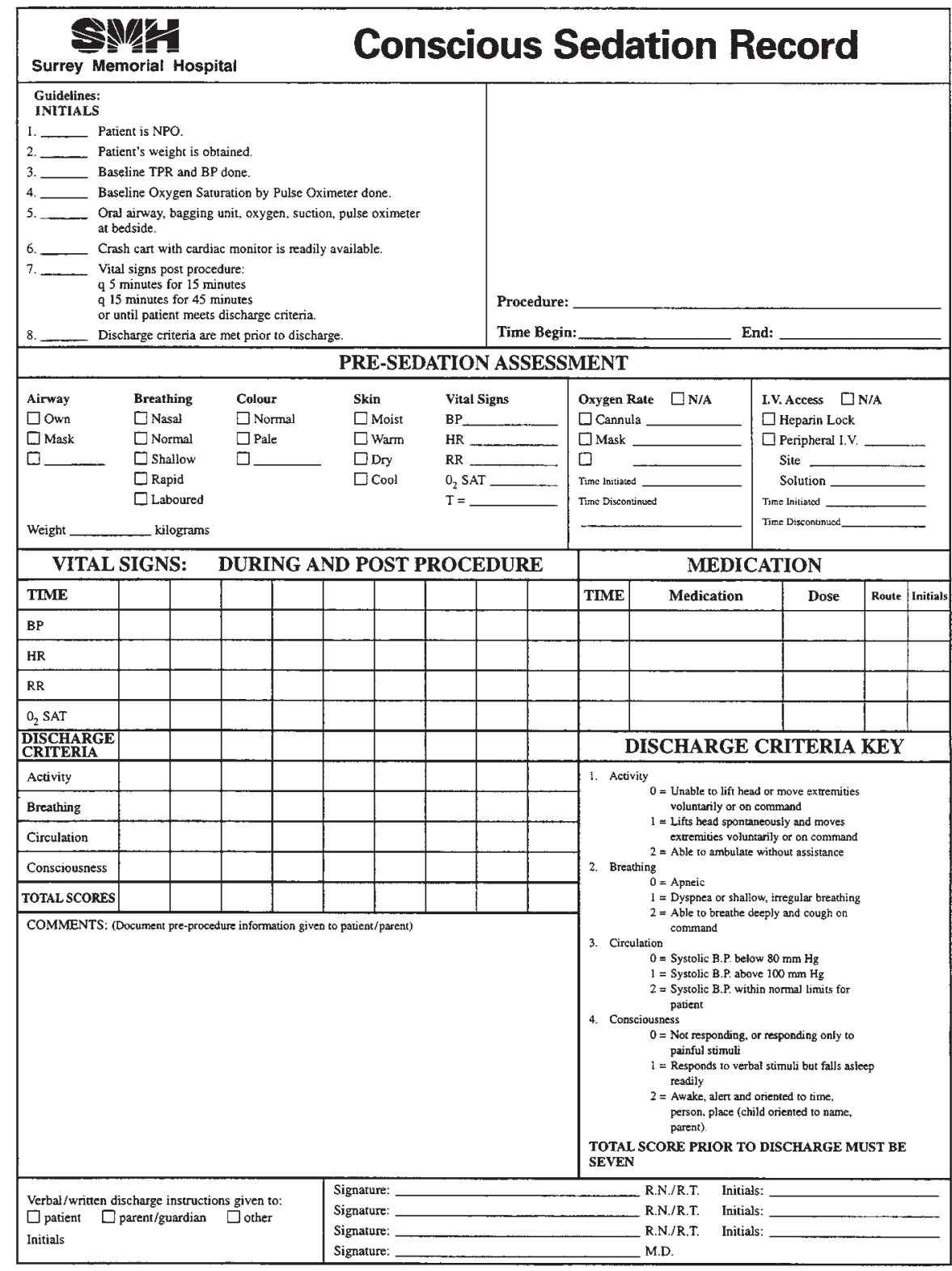

Fig. 2. Surrey Memorial Hospital procedural sedation record. 
or cardiorespiratory compromise were recorded, and no mortality or significant morbidity occurred. One 3-year-old female developed intractable screaming after receiving 2 $\mathrm{mg} / \mathrm{kg}$ IM ketamine for an elbow reduction, and one 4-yearold male experienced a visual hallucination within $24 \mathrm{~h}$ of receiving $5 \mathrm{mg} / \mathrm{kg}$ IM ketamine. The child told his parents he saw "Barney" (a purple dinosaur, popular child's cartoon character) coming out of the wall. He was not distressed by the hallucination and his parents were amused. The hallucination seemed to resolve within 1 minute.

Only 90 parents (54\%) could be reached for telephone follow-up. All expressed satisfaction with the procedure and indicated they would choose a similar technique if their child required a painful procedure in the future.

\begin{tabular}{|c|c|c|}
\hline Procedure & & $n$ \\
\hline Orthopedic & & $81(48.5 \%)$ \\
\hline Reduction, upper limb fracture & 36 & \\
\hline Reduction, lower limb fracture & 5 & \\
\hline Splinting of lower limb & 5 & \\
\hline Joint reduction & 35 & \\
\hline Wound repair & & $46(27.5 \%)$ \\
\hline Hand & 29 & \\
\hline Face & 17 & \\
\hline Foreign body removal & & $23(13.8 \%)$ \\
\hline Nares & 10 & \\
\hline Ear & 7 & \\
\hline Eye & 2 & \\
\hline Pharynx & 1 & \\
\hline Hand & 2 & \\
\hline Foot & 1 & \\
\hline Miscellaneous & & $17(10.2 \%)$ \\
\hline Dorsal slit of the penis & 5 & \\
\hline Ophthalmologic examination & 4 & \\
\hline Sedation for CT scan & 3 & \\
\hline Dental (splinting/extraction) & 2 & \\
\hline TMJ reduction & 1 & \\
\hline Fecal disimpaction & 1 & \\
\hline Rectal prolapse reduction & 1 & \\
\hline Total & & 167 \\
\hline
\end{tabular}

Table 3. Medications administered
\begin{tabular}{lccrr} 
Medication & Route & Dose $(\mathrm{mg} / \mathrm{kg})^{*}$ & \multicolumn{1}{l}{$n$} & $(\%)$ \\
\hline Ketamine & IM & $2.0-5.0$ & 129 & $(77)$ \\
Midazolam/fentanyl & IV/IV & $0.05-0.1 / 2.0-5.0^{*}$ & 28 & $(17)$ \\
Ketamine & IV & $0.5-1.0$ & 5 & $(3)$ \\
Ketamine/atropine & IM/IM & $2.0-5.0 / 0.01$ & 2 & $(1)$ \\
Ketamine/midazolam & IM/PO & $2.0-5.0 / 0.5$ & 2 & $(1)$ \\
Midazolam & IV & $0.05-0.1$ & 1 & $(1)$ \\
\hline${ }^{*}$ Fentanyl dose is in $\mathrm{mcg} / \mathrm{kg}$ & & & &
\end{tabular}

\section{Discussion}

Several authors have documented their experience with pediatric procedural sedation; ${ }^{11-20}$ however, this is the first published report describing the safety and effectiveness of pediatric sedation performed by emergency physicians in a Canadian ED.

In this study, males outnumbered females by a ratio of 1.6 to 1 , and 119 of 167 children ( $71 \%$ ) were under 4 years old. This is an age group in which vascular access is often difficult and painful - a stress to patient and family. In the current study, because of the availability of IM ketamine, 133 patients $(79.6 \%)$ underwent sedation without intravenous access. Our experience reflects that of other authors ${ }^{17,21}$ and suggests that an IV line is not necessary in otherwise healthy children undergoing ketamine procedural sedation.

If IV access is not available, physicians may administer oral, nasal or rectal agents; however, IM ketamine offers several advantages over these, including potent analgesia, amnesia and sedation, rapid onset, and preservation of ventilatory drive and airway protective reflexes. Most patients in this series received $4 \mathrm{mg} / \mathrm{kg}$ of IM ketamine (range 2-5 $\mathrm{mg} / \mathrm{kg}$ ). Sedation was adequate in $123(95 \%)$ of 129 patients after a single IM injection, but 6 patients - all undergoing orthopedic manipulation - required supplemental doses of 1 to $3 \mathrm{mg} / \mathrm{kg}$. Green and coworkers ${ }^{17}$ documented similar effectiveness, achieving adequate sedation in 398 of 431 patients after a single IM injection. Although some authors suggest that ketamine should be co-administered with benzodiazepines (to limit emergence agitation) or antisialogogues (to reduce salivation), our usual practice is to administer it without adjunctive medications. In this series, only 2 patients were co-administered atropine and 2 midazolam.

When IV access was available, our ED physicians tended to use a midazolam-fentanyl combination, titrated to effect. This combination provides potent sedation, analgesia and amnesia, and dual administration permits lower doses of each medication. Fentanyl and midazolam doses used in this study (Table 3) compare favourably with other published reports. ${ }^{7,11,15,22}$

The current controversy around procedural sedation focuses on safety and on whether or not emergency physicians are inducing general anesthesia in the ED. Commonly used agents like midazolam and fentanyl cause dose-related suppression of airway protective reflexes and ventilatory drive; therefore (particularly in cases requiring deeper sedation), they may provoke airway compromise, hypoventilation and hypotension. Clinicians employing these agents should be comfortable with airway management and familiar with the pertinent reversal agents, flumazenil and naloxone. 
In contrast, ketamine induces thalamoneocortical and limbic dissociation. In the low doses used in the ED, ketamine induces dissociative sedation, a state that falls short of general anesthesia because airway protective reflexes are preserved, ventilatory response to carbon dioxide is maintained, ${ }^{23}$ respirations are generally adequate and the eyes often remain open. Ketamine can, however, cause adverse effects, including hypersalivation, laryngospasm and apnea.

In a series of 1022 children who received IM ketamine, Green and coworkers ${ }^{17}$ reported 4 episodes of laryngospasm and 2 cases of transient apnea. Other authors have also reported rare episodes of laryngospasm and transient apnea, which may require suction or brief periods of assisted ventilation. ${ }^{17,19,24}$ In the current study, there were no episodes of apnea, hypotension or oxygen desaturation below $92 \%$. Nor were there episodes of laryngospasm, despite the fact that we rarely administer antisialogogues. Nevertheless, these issues remain a concern. Our sedation protocol prohibits ketamine use during procedures involving pharyngeal stimulation, and in patients receiving ketamine we avoid mechanical irritation of the laryngopharynx and discourage deep or aggressive suctioning. Further, we routinely place patients in the recovery position to allow gravity-assisted drainage of oral secretions.

While there was no mortality or significant morbidity in this study, many ketamine recipients experienced nausea and $12 \%$ of these children vomited. Vomiting occurred during the arousal phase and was easily treated with antiemetics. There were no episodes of clinical aspiration and all patients were discharged from the ED in satisfactory condition. Transient ataxia was also common during the recovery phase, but resolved quickly. One patient became agitated during emergence and another experienced a brief visual hallucination. Parents regarded these as minor events and, of those contacted for follow-up, all expressed a high degree of satisfaction with procedural sedation.

In this study, $82 \%$ of patients presented between 1500 and $0200 \mathrm{~h}$, a time when our operating rooms (OR) do primarily emergency cases and when lower priority cases like orthopedic manipulation and wound repair have poor OR access. If our patients were unable to have their procedures performed under ED sedation, most would encounter substantial treatment delays and endure prolonged pain, and many would be hospitalized for a day or more to accommodate their OR procedure. The ability to perform such procedures using ED procedural sedation therefore shortens time-to-treatment, limits unnecessary admissions, and reduces hospital, OR and anesthesiology costs. Apart from any potential cost savings, shorter treatment times limit inconvenience and fatigue for patients and caregivers, and reduce the need for caregivers to lose time from work.

\section{Limitations}

This study is large enough to demonstrate the effectiveness of ED pediatric sedation; however, because adverse outcomes after procedural sedation are exceedingly rare events, we cannot conclude that our sedation protocol has reduced the incidence of adverse outcomes; nor can we conclude that the likelihood of such events is zero.

Unfortunately, we achieved follow-up in only 54\% of cases. It is, therefore, conceivable, that we may have missed adverse symptoms or events that developed after ED discharge.

While we believe that ED procedural sedation shortens time-to-treatment and reduces both hospital- and patientborne costs, we did not perform a formal cost-effectiveness analysis to demonstrate these benefits.

\section{Conclusions}

Emergency physicians using a structured sedation protocol can safely perform ED pediatric procedural sedation. Where intravenous access is not already present, intramuscular ketamine, administered in the doses described, is a safe and effective agent. Minor adverse effects do occur but are easily manageable. Mortality and significant morbidity are extremely unlikely; however, advanced airway management skills are strongly recommended for physicians performing pediatric procedural sedation.

\section{References}

1. Chuang E, Wenner WJ Jr, Piccoli DA, Altschuler SM, Liacouras CA. Intravenous sedation in pediatric upper gastrointestinal endoscopy. Gastrointest Endosc 1995;42:156-60.

2. Lu DP, Lu GP, Reed JF III. Safety, efficacy, and acceptance of intramuscular sedation: assessment of 900 dental cases. Compendium 1994;15:1348.

3. Shane SA, Fuchs SM, Khine H. Efficacy of rectal midazolam for the sedation of preschool children undergoing laceration repair. Ann Emerg Med 1994;24:1065-73.

4. Pohlgeers AP, Friedland LR, Keegan-Jones L. Combination fentanyl and diazepam for pediatric conscious sedation. Acad Emerg Med 1995;2:879-83.

5. Alfonzo-Echeverri EC, Berg JH, Wild TW, Glass NL. Oral ketamine for pediatric outpatient dental surgery sedation. Pediatr Dent 1993;15:182-5.

6. Qureshi FA, Mellis PT, McFadden MA: Efficacy of oral ketamine for providing sedation and analgesia to children requiring laceration repair. Pediatr Emerg Care 1995;11:93-7.

7. Varela CD, Lorfing KC, Schmidt TL. Intravenous sedation for the closed reduction of fractures in children. J Bone Joint Surg Am 1995;77:340-5 .

8. Schechter NL, Weisman SJ, Rosenblum M, Bernstein B, Conard 
PL. The use of oral transmucosal fentanyl citrate for painful procedures in children. Pediatrics 1995;95:335-9.

9. Pruitt JW, Goldwasser MS, Sabol SR, Prstojevich SJ. Intramuscular ketamine, midazolam, and glycopyrrolate for pediatric sedation in the emergency department. J Oral Maxillofac Surg 1995;53:13-7.

10. Louon A, Reddy VG. Nasal midazolam and ketamine for pediatric sedation during computerized tomography. Acta Anaesthiol Scand 1994;38:259-61.

11. Parker RI, Mahan RA, Giugliano D, Parker MM. Efficacy and safety of intravenous midazolam and ketamine as sedation for therapeutic and diagnostic procedures in children. Pediatrics 1997; 99:427-31.

12. Dachs RJ, Innes GM. Intravenous ketamine sedation of pediatric patients in the emergency department. Ann Emerg Med 1997; 29:146-50

13. Roelofse JA, Joubert JJ, Roelofse PG. A double-blind randomized comparison of midazolam alone and midazolam combined with ketamine for sedation of pediatric dental patients. J Oral Maxillofac Surg 1996;54:838-44.

14. Petrack EM, Marx CM, Wright MS. Intramuscular ketamine is superior to meperidine, promethazine, and chlorpromazine for pediatric emergency department sedation. Arch Pediatr Adolesc Med 1996;150:676-81.

15. Graff KJ, Kennedy RM, Jaffe DM. Conscious sedation for pediatric orthopaedic emergencies. Pediatr Emerg Care 1996;12:31-5.

16. Riavis M, Laux-End R, Carvajal-Busslinger MI, Tschappeler H,
Bianchetti MG. Sedation with intravenous benzodiazepine and ketamine for renal biopsies. Pediatr Nephrol 1998;12:147-8 .

17. Green SM, Rothrock SG, Lynch EL, Ho M, Harris T, Hestdalen $\mathrm{R}$, et al. Intramuscular ketamine for pediatric conscious sedation in the emergency department: safety profile in 1,022 cases. Ann Emerg Med 1998;31:688-97.

18. Egelhoff JC, Ball WS Jr, Koch BL, Parks TD. Safety and efficacy of sedation in children using a structured sedation program. AJR Am J Roentgenol 1997;168:1259-62 .

19. Litman RS. Apnea and oxyhemoglobin desaturation after intramuscular ketamine administration in a 2-year-old child. Am J Emerg Med 1997;15:547-8.

20. Green SM, Rothrock SG. Intravenous ketamine for pediatric sedation in the emergency department, safety profile with 156 cases. Acad Emerg Med 1998;5:971-6.

21. Epstein FB. Ketamine dissociative sedation in pediatric emergency medical practice. Am J Emerg Med 1993;11:180-2.

22. Gremse DA, Kumar S, Sacks AI. Conscious sedation with highdose midazolam for pediatric gastrointestinal endoscopy. South Med J 1997;90:821-5 .

23. Soliman MG, Brinale GF, Kuster G. Response to hypercapnia under ketamine anaesthesia. Can Anesth Soc J 1975;22:486-94.

24. Smith JA, Santer LJ. Respiratory arrest following intrmuscular ketamine injection in a 4 year old child. Ann Emerg Med 1993;22:613-5.

Correspondence to: Urbain.Ip@sfvhr.hnet.bc.ca



